\title{
RENDIMIENTO ACADÉMICO Y SU RELACIÓN CON VARIABLES SOCIOEMOCIONALES EN ESTUDIANTES CHILENOS DE CONTEXTOS VULNERABLES
}

\author{
(ACADEMIC PERFORMANCE AND ITS RELATIONSHIP WITH SOCIO- \\ EMOTIONAL VARIABLES IN CHILEAN STUDENTS FROM VULNERABLE \\ CONTEXTS)
}

\author{
Angélica Vera Sagredo \\ Universidad Católica de la Santísima Concepción, Chile \\ Gamal Cerda Etchepare \\ Universidad de Concepción, Chile \\ Estíbaliz Aragón Mendizábal \\ Universidad de Cádiz, España \\ Carlos Pérez Wilson \\ Universidad de O'Higgins, Chile
}

DOI: $10.5944 / e d u c X X 1.28269$

\begin{abstract}
Cómo referenciar este artículo/How to reference this article:
Vera Sagredo, A., Cerda Etchepare, G., Aragón Mendizábal, E., y Pérez Wilson, C., (2021). Rendimiento académico y su relación con variables socioemocionales en estudiantes chilenos de contextos vulnerables. Educación XX1, 24(2), 375-398. https://doi.org/10.5944/educXX1.28269
\end{abstract}

Vera Sagredo, A., Cerda Etchepare, G., Aragón Mendizábal, E., \& Pérez Wilson, C., (2021). Academic performance and its relationship with socio-emotional variables in chilean students from vulnerable contexts. Educación XX1, 24(2), 375-398. https://doi.org/10.5944/educXX1.28269

\section{RESUMEN}

La declarada equidad educativa en el contexto chileno ha sido contrastada permanentemente como una dificultad para los estudiantes que se encuentran en contextos de alta vulnerabilidad social. En consecuencia, es procedente analizar factores no estructurales que puedan ser abordados por la comunidad educativa. Existe gran evidencia de la incidencia sobre el rendimiento educativo de ciertas variables como son los estilos atributivos, las estrategias de autorregulación, la convivencia escolar o el autoconcepto académico, pero un análisis específico y conjunto de estas variables para las realidades vulnerables podría entregar indicios a las comunidades educativas para priorizar e implementar oportunidades de mejora. El objetivo de este trabajo fue examinar el efecto conjunto de los factores mencionados respecto del logro académico de estudiantes de tercer y cuarto año medio de centros educativos chilenos vulnerables. En el estudio participaron 
alumnos de enseñanza secundaria de contextos de alta vulnerabilidad social $(\mathrm{N}=1498)$. Para la recogida de datos se emplearon cuatro encuestas tipo escala Likert que fueron aplicadas de forma masiva y on line. Mediante un modelo de ecuaciones estructurales, los resultados dan cuenta que el autoconcepto académico obtuvo la mayor significación estadística $(\beta=.22 ; p<.05)$, seguida de las estrategias de autorregulación $(\beta=.12 ; p<.05)$. También se observó que el factor de indisciplina obtuvo una asociación significativa, pero inversa ( $\beta=-$ $.22 ; p<.05)$ con las calificaciones de los estudiantes. Las correlaciones entre las variables también fueron significativas, observándose una fuerte correlación del rendimiento escolar con las estrategias de autorregulación y el autoconcepto académico. Las conclusiones se centran en la anticipación y prevención de situaciones de indisciplina, la promoción de un autoconcepto académico positivo y la implementación de programas específicos que contribuyan a la mejora de las estrategias de autorregulación, consideradas como prácticas o acciones situadas dentro del margen de actuación de la comunidad educativa.

\section{PALABRAS CLAVE}

Rendimiento académico, medidas emocionales, contextos vulnerables, modelo de ecuaciones estructurales

\section{ABSTRACT}

The declared educational equity in the Chilean context has been permanently contrasted as a difficulty presented by students who are in contexts of high social vulnerability. For this reason, it seems interesting to review other elements that do not belong to structural factors and that can be addressed by the educational community to support students who live in that reality. There is great evidence of the incidence of variables such as attributive styles, selfregulation strategies, school coexistence and academic self-concept regarding school performance, but a specific and joint analysis of these variables for vulnerable realities could provide clues to educational communities in order to prioritize and implement improvement opportunities. The objective of this work was to examine the joint effect of the aforementioned factors with respect to the academic achievement of middle third and fourth year students from vulnerable Chilean schools. The study involved secondary school students from highly socially vulnerable contexts $(\mathrm{N}=1498)$. Four Likert scale surveys were used for data collection, which were applied massively and online. Through the Structural Equations Model, results show that academic self-concept obtained the highest statistical significance $(\beta=.22 ; p<.05)$, followed by self-regulation strategies $(\beta=.12$; $\mathrm{p}<.05)$. It was also observed that the indiscipline factor obtained a significant but inverse association $(\beta=-.22 ; \mathrm{p}<.05)$ with the grades of the general average of the students. The correlations between the variables were also significant, showing that school performance would have a stronger and positive correlation with 
self-regulation strategies and academic self-concept. The conclusions focus on the anticipation and prevention of situations of indiscipline, the promotion of a positive academic self-concept and the implementation of specific programs that contribute to improvement in self-regulation strategies; considered as practices or actions located within the scope of action of the educational community.

\section{KEY WORDS}

Academic achievement, affective measures, disadvantaged environment, structural equation models

\section{INTRODUCCIÓN}

Una de las dificultades que presenta el sistema educativo chileno está relacionado con la equidad, entendida esta como el acceso igualitario a una educación de calidad, que en el caso particular de los estudiantes que asisten a centros educativos de alta vulnerabilidad social se expresa en bajos logros de aprendizaje (Vengas et al., 2016). Distintas investigaciones dan cuenta de que un estudiante que se encuentra en un contexto social vulnerable, podría estar expuesto a un entorno sociocultural empobrecido de estímulos y de apoyos familiares, lo que repercutiría en sus logros académicos (Villalta, 2010; Villalta \& Saavedra, 2012). Si bien hay reportadas algunas evidencias testimoniales de éxito, estas obedecen a factores muy difíciles de replicar, como la vida escolar, los eventos significativos previos, las relaciones con sus compañeros y el apoyo de las familias y profesores (Morales \& Morales, 2019).

La literatura confirma desde hace años una alta estratificación social en cuanto al itinerario de logros académicos en Chile (Orellana et al., 2019; Rosas \& Santa Cruz, 2013). En tal escenario, y tratando de disminuir el rol importante que tiene la estratificación sociocultural del estudiantado chileno en sus logros académicos, resulta fundamental examinar otros factores que podrían estar asociados a sus resultados, ya que el conocimiento y la comprensión de estos posibilitaría implementar mejoras pertinentes y medidas remediales basadas en aquellas variables o factores susceptibles de intervenir en el contexto escolar (Cerda \& Vera-Sagredo, 2019).

Uno de estos factores está dado por las atribuciones causales, haciendo referencia a las causas a través de las cuales las personas explicarían sus fracasos y éxitos escolares. Desde los pioneros trabajos de Weiner (1979), distintos autores han venido dando cuenta de que estas tendrían importantes consecuencias psicológicas, tanto a nivel emocional, cognitivo y motivacional (Amata et al., 2017; Delgado et al., 2018). A nivel emocional, las variables causales se relacionarían con sentimientos acerca del yo, como la autoestima o el orgullo; a nivel cognitivo, estas influirían en el cambio o mantenimiento 
de las expectativas de logros futuros; y a nivel motivacional, mediarían en posibilitar, dinamizar u obstaculizar sus acciones. En este constructo teórico las dimensiones atribucionales serían esquematizadas en función de tres parámetros: en primer término, según el nivel de causalidad "interna-externa"; en segundo término, relacionada con la estabilidad en el tiempo "estable-inestable"; y en tercer término, según la función de controlabilidad "controlable-incontrolable" (Weiner, 1979). Desde esta perspectiva, se podrían atribuir los éxitos o fracasos a cuatro causas principales: el esfuerzo de la persona, la dificultad de la tarea, la suerte y la capacidad del individuo. En este contexto, los estudiantes con locus de control interno creen que son responsables de su conducta y de sus resultados, atribuyendo sus logros a factores como la capacidad o esfuerzo. En cambio, los estudiantes con locus de control externo creen que su desempeño se debe a la suerte, a la dificultad de la tarea u otras circunstancias que se encuentran fuera de su control (Padua, 2019).

Las consecuencias derivadas respecto a las atribuciones realizadas por los estudiantes, podrían conformar un estilo atribucional que puede favorecer o desfavorecer su aprendizaje (Barca-Lozano et al., 2019; Lagos et al., 2016). Algunas investigaciones han corroborado que los estudiantes con patrones atribucionales básicamente externos (fracaso por el profesor, fracaso por la falta de esfuerzo, fracaso por la falta de habilidad) obtendrían resultados académicos bajos (Fernández, 2017; Ramudo et al., 2017; Ramudo, Barca et al., 2020; Rodríguez-Rodríguez \& Guzmán-Rosquete, 2019). Por ejemplo, el estudio de González-Berruga (2020) da cuenta de que los estudiantes con fracaso escolar, descritos como aquellos que han repetido curso, atribuirían su fracaso al docente señalando que es el profesor el que no motiva al aprendizaje. De igual forma, otros estudios arrojarían evidencia en esta línea, mostrando cómo los estudiantes atribuyen sus malos resultados, entre otros aspectos, al docente, considerando que no se encuentra preparado para dictar una clase (Cerda et al., 2019; Cerda \& Vera-Sagredo, 2019; Ibarra, 2019). En cambio, los estudiantes que tienen buen rendimiento académico se caracterizarían por atribuir sus éxitos y fracasos a su propia capacidad y esfuerzo. Desde lo señalado se aprecia que el conocimiento que se tenga de los estilos atribucionales permitiría comprender la motivación de los estudiantes cuando se enfrentan a un ambiente de aprendizaje, cuáles son las causas a las que asocian sus logros académicos, desde donde se podría inferir cuáles podrían ser las repercusiones futuras sobre sus expectativas y probables respuestas de tipo afectivas y conductuales (Amata et al. 2017; Jurado et al., 2016; Ruiz, 2017).

Del mismo modo, también ha sido reportado en bastantes investigaciones el hecho de que las estrategias de autorregulación pueden repercutir en el rendimiento académico de los estudiantes (Martínez \& Medina, 2019; Martínez \& Valiente, 2019). Este factor ha sido definido por distintos autores como habilidades complejas que integran pensamientos y comportamientos que facilitan la adquisición, almacenamiento y utilización de información desde la propia experiencia a través de componentes cognitivos, metacognitivos y 
afectivo-emocionales (García-Ripa et al., 2016). El uso de estas estrategias es importante a la hora de experimentar nuevos conocimientos, ya que permiten actuar estratégicamente al utilizar procedimientos, habilidades y técnicas eficaces para aprender (Suárez et al., 2015). En este sentido, el estudiante será capaz de desarrollar una conciencia metacognitiva que le permita regular las acciones a realizar, conociendo de qué forma aprende, reconociendo sus propias dificultades, y sistematizando las acciones a través de procesos de planificación, supervisión y revisión de sus prácticas para el cumplimiento de sus metas (Arias \& Aparicio, 2020; Valle et al., 2009). Además, estas estrategias permiten la supervisión del avance en el proceso de aprendizaje del estudiante y la evaluación constante de las acciones que utilizan para enfrentar sus retos académicos; esto implica un proceso de ensayo y error, que busca dar soluciones a las tareas (Castrillón et al., 2020).

Otra variable que se asocia a los niveles de logro de los estudiantes de la educación formal corresponde a los factores de convivencia escolar (Cerda et al., 2019; Wang et al., 2014). En el contexto educativo chileno, el interés por este factor emerge al observar que es uno de los indicadores que presenta una asociación más fuerte con los aprendizajes de los estudiantes. El Sistema Nacional de Evaluación de Resultados de Aprendizaje (SIMCE) (Educación 2020, 2015) informa de una diferencia de hasta 42 puntos entre centros educativos con alto y bajo clima escolar. Además, este factor es especialmente preocupante en centros educativos de contextos vulnerables, ya que son los que muestran una menor valoración del clima de convivencia escolar (Agencia de la Calidad, 2015). Para el Ministerio de Educación de Chile, las relaciones interpersonales que se producen en la unidad educativa son un componente sustancial de la cultura escolar, donde se manifiestan los estilos de relaciones que se dan entre los actores educativos, la manera de abordar las situaciones de conflictos y la posibilidad de expresarse que tienen los estudiantes, apoderados y miembros de los centros educativos (MINEDUC, 2015).

Asimismo, el concepto de convivencia escolar involucra una serie de interrelaciones y dinámicas entre los diferentes agentes educativos, incidiendo de múltiples maneras y reflejándose en muchos aspectos, como la desidia docente, indisciplina, red social de iguales, entre otros factores (Cerda, Salazar, Guzmán \& Narváez, 2018). Específicamente, la dimensión de indisciplina escolar percibida tiene consecuencias importantes en los procesos de enseñanza, y por tanto, implicaciones en el rendimiento académico (Cerda et al., 2018; Krskova \& Baumann, 2017), reportándose además efectos específicos en rendimientos disciplinares como el deporte (Claver et al., 2020) o asignaturas STEM (Ibrahim \& Johnson, 2020). Investigaciones recientes darían cuenta de que existirían efectos inversos entre la disciplina percibida y el rendimiento académico de los estudiantes (Bravo et al., 2018; Cerda et al., 2019).

Finalmente, dentro del conjunto de variables afectivas y actitudinales que pueden afectar al rendimiento escolar, está el autoconcepto, específicamente, 
el autoconcepto académico. El autoconcepto académico sería la percepción y evaluación que tiene un sujeto de su propia competencia académica (Cazalla-Luna \& Molero, 2015). Gran parte de las dificultades que presentan los adolescentes respecto del rendimiento académico está relacionado con la autopercepción que estos tengan sobre sí mismos, lo cual se ve potenciado por las particularidades propias de la adolescencia (Gonzálvez et al., 2016).

Distintas investigaciones constatan la relevancia del autoconcepto académico. Un estudiante autopercibido como competente, valorará el desafío de la tarea y favorecerá sentimientos de autoeficacia y de control sobre su propia conducta, sintiéndose por tanto directamente responsable de sus logros y aprendizajes (Morales, 2017). En general, las investigaciones revelan una relación positiva entre autoconcepto académico y el logro académico (Chacón \& Huertas, 2017; Kumar \& Choudhuri, 2017). El autoconcepto tiene efectos positivos en la relación maestro-alumno, la relación con los compañeros, la participación en actividades y el clima escolar (Huang, Yu \& Wu, 2019). Esto último es relevante, pues aquellos estudiantes con una percepción más alta de un clima escolar positivo exhiben un mejor rendimiento académico (Daily et al., 2020). Hay que considerar que el autoconcepto académico está vinculado, no solo al compromiso con la tarea, sino también al modo de afrontar los problemas, y las relaciones con los iguales y el profesorado (González et al., 2012). Este último aspecto se relaciona con la indisciplina y las conductas disruptivas en el aula, ya que parece ser que una relación profesor-alumno basada en el uso constante de etiquetas negativas por parte de los profesores repercutiría en el autoconcepto, especialmente, de sujetos procedentes de contextos vulnerables (Ngwokabuenui, 2015).

En función de estos antecedentes se postula examinar el rol y la relación de las variables autoconcepto académico, estrategias de supervisión, estilos atributivos de fracaso y percepción de indisciplina dentro del dominio de la convivencia escolar con respecto al rendimiento académico. El objetivo fundamental del estudio es examinar el efecto conjunto de los factores mencionados relacionados con el logro académico de estudiantes de tercer y cuarto año medio de centros educativos chilenos vulnerables.

De acuerdo con el marco teórico analizado, el modelo hipotetizado postula a priori encontrar una relación positiva y significativa entre el autoconcepto académico y la estrategia de supervisión respecto del desempeño académico e inversamente, una relación negativa y significativa del estilo atributivo de fracaso y la percepción de indisciplina en el desempeño de los estudiantes. Del mismo modo, en cuanto a efectos mediados, se hipotetiza que el autoconcepto académico tendrá una relación intermediada por la percepción de indisciplina, y también con respecto a las posibles atribuciones de fracaso, las cuales, a su vez, interactúan con la posibilidad de llevar a cabo estrategias de supervisión de estudios que impacten positivamente sobre el rendimiento, generando un efecto indirecto de todas ellas sobre el desempeño académico general de los estudiantes. Las diversas relaciones se describen en la Figura 1. 
Figura 1

Modelo hipotetizado a partir la fundamentación teórica

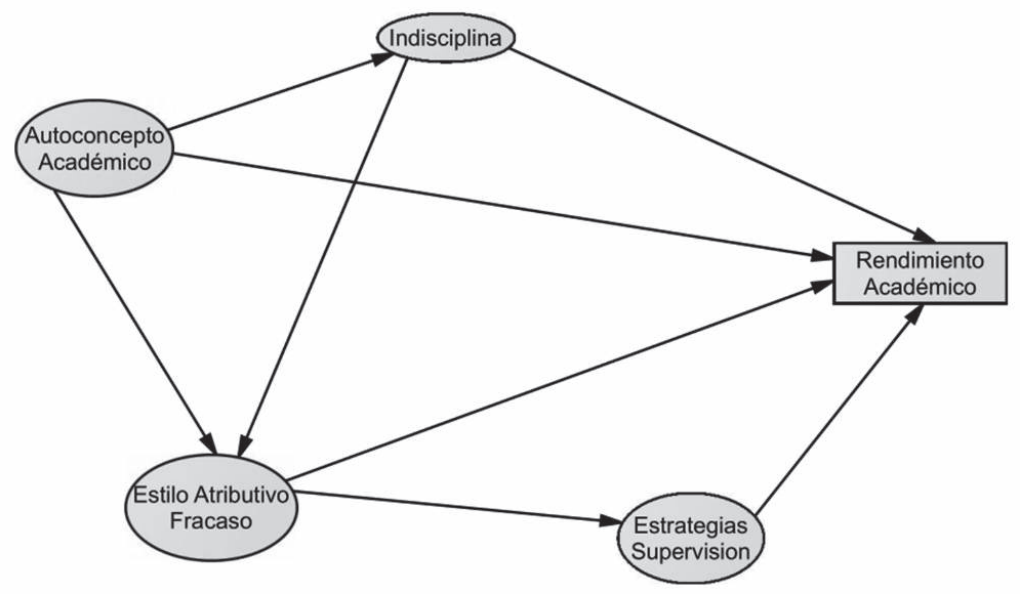

Fuente: Elaboración propia

\section{METODOLOGÍA}

\section{Muestra}

La muestra del estudio está conformada por 1498 estudiantes de educación secundaria de 22 centros educativos públicos con altos índices de vulnerabilidad escolar (IVE) pertenecientes a la región del Biobío, Chile.

El Índice de Vulnerabilidad Escolar (IVE), es un parámetro calculado anualmente por la Junta Nacional de Auxilio Escolar y Becas (JUNAEB) del Gobierno de Chile, que se expresa en términos porcentuales. Este índice refleja el grado de vulnerabilidad, calculado según factores socioeconómicos, indicadores de rendimiento y asistencia de los estudiantes (Educación 2020, s.f.). Para calcular este índice, se clasifica a los estudiantes en 3 niveles, denominados prioridades, según condiciones de pobreza y riesgo de fracaso escolar. La primera prioridad corresponde a alumnos en condición de extrema pobreza, mientras que la segunda y tercera prioridad se construye sobre métricas de riesgo de fracaso escolar. Así, el índice IVE se determina como:

$$
I V E=\frac{\left(P_{1}+P_{2}+P_{3}\right)}{\text { Matrícula Establecimiento }} \times 100 \%
$$

En la expresión anterior, se incluye la cantidad de estudiantes del centro educativo que son clasificados en los niveles o prioridades 1, 2 y 3 respectivamente. 
De acuerdo a esta expresión, un mayor porcentaje implica índice de vulnerabilidad más elevado. Si bien no hay baremos específicos para definir el concepto de alta vulnerabilidad, en general se suele considerar al conjunto de centros educativos del quinto quintil (80\% hacia arriba).

En cuanto al procedimiento de muestreo, por tratarse de un estudio exploratorio, se realizó un muestreo no probabilístico de tipo intencional en 22 establecimientos de alta vulnerabilidad, que corresponden a un $10 \%$ del total de liceos con IVE calculado en la región donde se enmarca el estudio. Para ello se accedió a los cursos de tercero y cuarto año de enseñanza media de dichos centros educativos, que corresponden a los dos últimos años de escolarización, equivalentes a primero y segundo de bachillerato o al nivel de Educación Secundaria Superior de acuerdo a la Clasificación Internacional Normalizada de la Educación. La solicitud de participación fue realizada al total de estudiantes de estos cursos, registrando una participación superior al 93\% del total de los alumnos en cada centro educativo. Del total de estudiantes que formaron parte de la muestra, $802(53.3 \%)$ eran mujeres y $696(46.5 \%)$ hombres. Los participantes tienen entre 16 y 22 años de edad $(M=17.19 ; D E=.976)$. Respecto a la distribución por curso, el 49.9\% (748 estudiantes) pertenecen a tercer año medio y el 50.1\% (750 estudiantes) a cuarto año medio. Los casos de edades altas, sobre $18(\mathrm{~N}=143)$, corresponden a un grupo pequeño de casos de estudiantes de establecimientos educativos adscritos al programa de Educación de Personas Jóvenes y Adultas (MINEDUC, 2020).

\section{Instrumentos}

Cada una de las variables socioemocionales fueron medidas considerando un instrumento específico, basados en escalas tipo Likert, que son descritos a continuación. El detalle de sus ítems y dimensiones son presentados en la Tabla 1.

Estilos atributivos frente al fracaso académico. Se evalúan tres factores de los estilos atributivos académicos que forman parte de la Escala de Estilos Atributivos (EAT) (Alonso \& Sánchez, 1992). Los factores son obtenidos de la escala EAT validada para estudiantes chilenos (Vera, Cerda y Melipillán, 2020, en prensa). Los índices de ajuste del análisis factorial confirmatorio para la muestra es adecuado, exhibiendo índices de bondad de ajuste pertinentes: chi cuadrado Satorra-Bentler, SB $\chi 2(74)=238.32, p<.001$; Comparative Fit Index, CFI= .980 (Robust CFI=.972); Bentler-Bonett Non Normed Fit NNFIT= .976; Root Mean Square Error of Approximation, RMSEA = .039, IC (.033.044). El índice de fiabilidad también resulta apropiado, mostrando un valor de Alpha de Cronbach, $\alpha=.685$, con un índice de consistencia interna máxima ponderada de .81 .

Estrategia de autorregulación de supervisión. Se examina a través del factor de supervisión de la escala de estrategias de autorregulación (Hernández \& 
García, 1995). Los índices de ajuste del análisis factorial confirmatorio muestran ser adecuados a este factor: SB $\chi 2(35)=145.15, p<.001, \mathrm{NNFIT}=.987, \mathrm{CFI}=$ .990 (Rubust CFI = .986); RMSEA = .046, IC (.034-.054). El índice de fiabilidad resultó apropiado $(\alpha=.88)$, al igual que el índice de consistencia interna máxima ponderada (.90).

Indisciplina Percibida. Se utiliza la dimensión de la Escala de Convivencia Escolar (Del Rey, Casas \& Ortega, 2017), la cual ha corroborado su estructura de invarianza factorial en la población escolar chilena y española (Elipe et al., 2018). El análisis factorial confirmatorio muestra medidas de bondad de ajuste adecuadas: $\mathrm{SB} \chi 2(2)=3.513, p<.017, \mathrm{CFI}=.997$ (Robust $\mathrm{CFI}=.992)$, NNFIT=.990; RMSEA $=.022$, IC (.000-.061). El índice de fiabilidad resultó apropiado $(\bigotimes=.57)$, al igual que el índice de consistencia interna máxima ponderada (.80).

Autoconcepto Académico: Se utilizó específicamente la dimensión Autoconcepto Intelectual del Cuestionario de Autoconcepto de Garley (García, 2001). Los índices de bondad de ajuste del análisis factorial confirmatorio muestran que el ajuste del modelo es adecuado: SB $\chi 2(20)=1799.512, p<$ $.017, \mathrm{CFI}=.973$ (Robust $\mathrm{CFI}=.969), \mathrm{NNFIT}=.962$; RMSEA $=.073$, IC $(.063-$ $.083)$. El índice de fiabilidad resultó apropiado $(\alpha=.88)$, al igual que el índice de consistencia interna máxima ponderada (.87).

Finalmente, para la variable rendimiento académico, se utilizó el promedio general de calificaciones correspondientes al año lectivo actual que cursan los estudiantes, estandarizadas y transformadas a puntajes T. Las calificaciones fueron facilitadas por los propios centros educativos, y extraídas directamente de las actas que figuran en los documentos oficiales.

Tabla 1

Dimensiones e items de las distintas escalas utilizadas en el estudio

Dimensión

\section{Escala Estilos atributivos frente al fracaso académico}

A menudo, si he tenido malas notas ha sido porque los profesores no me han explicado bien las materias.

Atribución fracaso académico por el profesor. 5 ítems, graduación: 5 (máximo acuerdo) a 1 (máximo desacuerdo).
Con frecuencia mis malas notas se deben a que el profesor no está bien preparado para enseñarme.

Si he tenido malas notas a menudo ha sido porque el profesor explicaba mal.

Normalmente, si he sacado malas notas ha sido porque el profesor era un tacaño al puntuar.

Mis malas notas se han debido, con frecuencia, a que el profesor puntuaba muy bajo. 
Dimensión

\begin{tabular}{l}
\hline \\
Atribución del fracaso \\
académico a la falta \\
de esfuerzo. 6 ítems, \\
graduación: 5 (máximo \\
acuerdo) a 1 (máximo \\
desacuerdo).
\end{tabular}

Atribución del fracaso a la falta de habilidad. 3 ítems, graduación: 5 (máximo acuerdo) a 1 (máximo desacuerdo).

\section{ítems}

Las bajas notas que he obtenido han sido, sobre todo, porque no me he esforzado lo suficiente.

Normalmente, si recibo una mala nota en una asignatura es porque no he estudiado lo suficiente.

En general, las notas bajas han reflejado mi poco trabajo y esfuerzo.

Las malas notas significan para mí que no he trabajado con suficiente empeño.

Si alguna vez he obtenido bajas calificaciones se ha debido a falta de esfuerzo.

Si las notas que saco no son tan buenas como esperaba, normalmente pienso que se debe a mi falta de esfuerzo.

Si sacase malas notas dudaría de mi inteligencia.

Cuando saco malas notas pienso que no soy inteligente.

Si sacase malas notas pensaría que no tengo el talento necesario para comprender esas asignaturas.

\section{Estrategia de autorregulación de supervisión}

Si hay algo que no entiendo o no sé hacer, procuro no seguir adelante hasta lograr resolverlo.

Algunas veces, si no entiendo o no sé hacer algo, procuro seguir adelante para ver si lo siguiente me puede aclarar lo anterior.

Cuando se me presenta algún problema o cuestión, procuro siempre buscar, prioritariamente, dentro de mí la solución.

Cuando no logro resolver un problema por mí mismo, acudo a otras personas o libros que me lo aclaren.

Supervisión. 10 ítems, Mientras estudio, suelo estar continuamente revisando lo graduación: 5 (máximo acuerdo) a 1 (máximo que está flojo o en lo que fallo, para amarrarlo bien.

Cuando estudio, o resuelvo un problema, procuro consultar la opinión de otros para ver si lo que estoy haciendo es correcto.

Cuando he terminado de estudiar, tengo la costumbre de hacer una revisión de todo para ver si tengo algún fallo.

Cuando he terminado de estudiar, procuro amarrar y consolidar los puntos que considero más débiles.

Cuando he terminado de estudiar, procuro relacionar lo estudiado con el examen o con lo que el profesor me pide.

Cuando he estudiado un tema, y luego pasa un tiempo, trato de volverlo a repasar o refrescarlo en la memoria antes de una prueba o examen. 


\begin{tabular}{|c|c|}
\hline Dimensión & ítems \\
\hline \multicolumn{2}{|l|}{ Indisciplina Percibida } \\
\hline \multirow{4}{*}{$\begin{array}{l}\text { Indisciplina. } 4 \text { ítems, } \\
\text { graduación: } 5 \text { (máximo } \\
\text { acuerdo) a } 1 \text { (máximo } \\
\text { desacuerdo). }\end{array}$} & Solo cumplo las normas que me convienen. \\
\hline & Me han castigado. \\
\hline & Interrumpo la clase porque me aburro. \\
\hline & Me aburro en el colegio. \\
\hline \multicolumn{2}{|c|}{ Autoconcepto Académico } \\
\hline \multirow{8}{*}{$\begin{array}{l}\text { Autoconcepto } \\
\text { Intelectual. } 8 \text { ítems, } \\
\text { graduación: } 5 \text { (máximo } \\
\text { acuerdo) a } 1 \text { (máximo } \\
\text { desacuerdo). }\end{array}$} & Soy lento (o lenta) haciendo mi trabajo escolar. \\
\hline & Soy un buen lector (o buena lectora). \\
\hline & Puedo recordar fácilmente las cosas. \\
\hline & Respondo bien en clase. \\
\hline & Termino rápidamente mi trabajo escolar. \\
\hline & Creo que soy inteligente. \\
\hline & Soy lista (o listo). \\
\hline & Hago bien mi trabajo intelectual. \\
\hline
\end{tabular}

\section{Procedimiento}

El procedimiento de aplicación de los instrumentos se realizó de forma masiva al conjunto de estudiantes de la muestra, requiriendo sus asentimientos y la firma del consentimiento por parte de sus padres o tutores en el caso de ser menores de edad. Los instrumentos se aplicaron en versión online, respetando su estructura original. Previamente a esta modalidad de aplicación, se realizó un diagnóstico de las condiciones tecnológicas de cada centro educativo, constatando que todos contaban con laboratorios de computación y acceso a internet. Los horarios de aplicación fueron coordinados con los directores y profesores de tecnología, para no perjudicarlos en sus obligaciones escolares.

Respecto de los lineamientos éticos propios del estudio, los protocolos de consentimiento y asentimiento fueron primeramente visados por el Comité de Ética Institucional de la Universidad de Concepción, Chile, el cual verificó su pertinencia y apego a los lineamientos de investigación en ciencias sociales. Del mismo modo, a los participantes y padres o tutores responsables, se les explicó la naturaleza del estudio, y se respondieron dudas sobre alcances, confidencialidad y resultados a obtener. 


\section{Análisis de datos}

Primeramente se utilizó para cada una de las variables la puntuación derivada de la suma de los ítems de los instrumentos con los que se evaluaron. Seguidamente se aplicó el coeficiente de correlación bivariado de Pearson, para examinar las asociaciones de todas las variables en estudio y el rendimiento académico. Finalmente, con el propósito de analizar la relación compleja e interdependiente de las variables en estudio, en virtud del modelo hipotetizado se realizó un procedimiento confirmatorio mediante Ecuaciones Estructurales, que permite confrontar el conocimiento a priori e hipótesis con los datos empíricos.

Para el conjunto de los análisis se emplearon los paquetes estadísticos SPSS v. 23.0 y el programa EQS® v. 6.2.

\section{RESULTADOS}

La Tabla 2 presenta la matriz de correlaciones de todas las variables consideradas.

Tabla 2

Media (M), desviación estándar (DE) y correlaciones de Pearson de las variables incluidas en el modelo

\begin{tabular}{|c|c|c|c|c|c|c|c|c|}
\hline & M & DE & 2 & 3 & 4 & 5 & 6 & 7 \\
\hline $\begin{array}{l}\text { 1.Autoconcepto } \\
\text { académico }\end{array}$ & 22.47 & 3.75 & $-.110^{* *}$ & $.421^{* *}$ & $-.054^{*}$ & .050 & $-.215^{* *}$ & $.235^{* *}$ \\
\hline 2. Indisciplina & 4.38 & 1.35 & 1 & $-.255^{* *}$ & $-.233^{* * *}$ & $-.064^{*}$ & $.119^{* * *}$ & $-.215^{* * *}$ \\
\hline $\begin{array}{l}\text { 3.Estrategia de } \\
\text { autorregulación } \\
\text { de Supervisión }\end{array}$ & 35.02 & 6.82 & & 1 & $-.070^{* *}$ & $.130^{* *}$ & -.039 & $.245^{* *}$ \\
\hline $\begin{array}{l}\text { 4. Atribución de } \\
\text { fracaso por el } \\
\text { profesor }\end{array}$ & 7.56 & 2.61 & & & 1 & $-220^{* *}$ & $.269^{* * *}$ & $-.173^{* *}$ \\
\hline $\begin{array}{l}\text { 5. Atribución de } \\
\text { fracaso por falta } \\
\text { de esfuerzo }\end{array}$ & 14.51 & 2.63 & & & & 1 & $.058^{*}$ & $.056^{*}$ \\
\hline $\begin{array}{l}\text { 6. Atribución de } \\
\text { fracaso por falta } \\
\text { de habilidad }\end{array}$ & 5.02 & 1.83 & & & & & 1 & $-.126^{* * *}$ \\
\hline $\begin{array}{l}\text { 7. Rendimiento } \\
\text { Académico }\end{array}$ & 50.05 & 13.96 & & & & & & 1 \\
\hline
\end{tabular}


Como se muestra en la Tabla 2, en general aquellos estudiantes que tienen un mejor autoconcepto académico, tienden a tener un mejor rendimiento $(r=.235 ; p<.01)$. De modo similar, los sujetos que manifiestan tener mayor uso de estrategias de autorregulación de supervisión, alcanzan mejores calificaciones académicas $(r=.245 ; p<.01)$. De modo inverso, se observa que aquellos estudiantes que perciben o vivencian mayores situaciones de indisciplina dentro de los centros educativos, tienden a alcanzar peores desempeños académicos $(r=-.215$; $p<.01)$. El mismo tipo de relación negativa y estadísticamente significativa se observa entre las atribuciones de fracaso basado en el profesor $(r=-.173 ; p<.01)$ y las atribuciones de fracaso basado en la falta de habilidad con el rendimiento académico $(r=-.126 ; p<.01)$.

Por otra parte, se deben destacar también las asociaciones positivas y estadísticamente significativas entre el autoconcepto académico de los estudiantes y el uso de estrategias de supervisión $(r=.421 ; p<.01)$, y la relación entre la atribución de fracaso basada en el profesor y aquella atribución de fracaso basada en la falta de habilidad $(r=.269 ; p<.01)$. De un modo contrario, se observa que existe una relación negativa entre el autoconcepto académico y la percepción de indisciplina $(r=-.110 ; p<.01)$ y entre esta última y la estrategia de autorregulación de supervisión $(r=-.255 ; p<.01)$.

El modelo de ecuaciones estructurales examinó las relaciones entre las diferentes variables latentes y sus indicadores, que podrían estar influyendo en el rendimiento académico de los estudiantes. Se aplicó el modelo de máxima verosimilitud, los índices de curtosis y asimetría para cada una de las variables resultan adecuados, dado que ninguno de ellos es superior al valor de \pm 2 , además el coeficiente de curtosis multivariante de Mardia es de 158.55, el cual resulta inferior al producto $p(p+2)$, siendo $p$ el número de variables observadas (Mardia, Kent \& Bibby, 2003). La estimación de los parámetros resultó estadísticamente significativa y las cargas factoriales se encontraron con un ajuste adecuado de los datos, $\mathrm{SB} \chi 2(290)=1096.92, p<.001 ; \mathrm{NNFI}=.896, \mathrm{CFI}=.907 ; \mathrm{y} \mathrm{RMSEA}=.043$ IC (.040-.046).

En la Figura 2 se puede observar el resultado del modelo confirmatorio, que considera las relaciones entre las variables objeto de estudio. 
Figura 2

Modelo de interacción propuesto para las diversas variables respecto al rendimiento académico

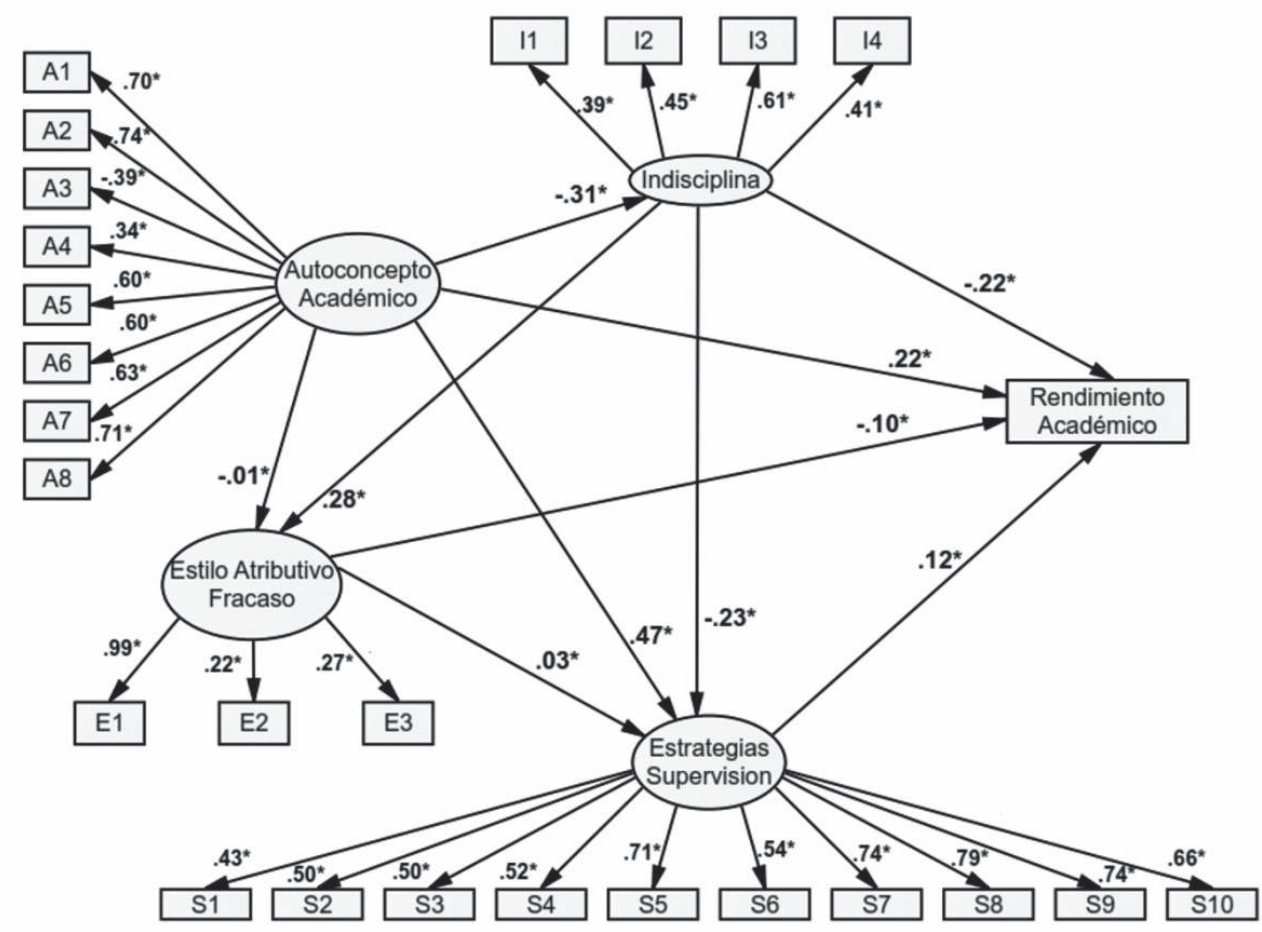

Fuente: Elaboración propia

A partir del análisis de los coeficientes de regresión estandarizados del modelo, se evidencia una relación positiva y significativa entre el autoconcepto académico y el rendimiento académico $(\beta=.22 ; p<.05)$. Respecto de esta variable, se observa que existe igualmente una mediación positiva y significativa con la estrategia de autorregulación de supervisión $(\beta=.47 ; p<.05)$, y también una mediación negativa y significativa con la percepción de indisciplina que tienen los estudiantes $(\beta=-.31 ; p<.05)$. Existe, además, una relación positiva entre la estrategia de autorregulación y el desempeño de los estudiantes $(\beta=.12 ; p<.05)$ y se evidencia una relación negativa y significativa entre la percepción de indisciplina y las calificaciones obtenidas por los sujetos $(\beta=-.22 ; p<.05)$. Del mismo modo, se observa una relación negativa y significativa entre el estilo atributivo de fracaso y la nota media $(\beta=-.10 ; p<.05)$. Por otra parte, se observa una relación mediada positiva y significativa de la percepción de indisciplina y el estilo atributivo de fracaso $(\beta=.28 ; p<.05)$, como también, una relación negativa y significativa de esta variable con la estrategia de autorregulación de supervisión $(\beta=-.23 ; p<.05)$. El modelo explica un $22.2 \%$ de la varianza del rendimiento académico. 


\section{DISCUSIÓN Y CONCLUSIONES}

El objetivo principal de este estudio ha sido analizar el efecto conjunto de una serie de variables socioemocionales respecto del rendimiento académico, para lo cual se ha propuesto un modelo basado en el análisis de ecuaciones estructurales que ha permitido explicar el impacto y peso estadístico de diferentes factores en el desempeño académico de estudiantes chilenos de nivel secundario, pertenecientes a centros educativos vulnerables.

Para cumplir con el objetivo se propuso un modelo teórico inicial basado en los distintos antecedentes teóricos y empíricos que justificaban las relaciones entre estas variables y los logros académicos. El modelo confirmatorio obtenido ha mostrado otras interacciones entre las variables no consideradas inicialmente, con las cuales se obtienen adecuados índices de bondad de ajuste. El autoconcepto académico surge como aquel factor con mayor significación estadística, seguido de la estrategia de autorregulación supervisión. Se destaca además la influencia negativa de la indisciplina y de las atribuciones de fracaso escolar por causas externas respecto al rendimiento académico.

Se observa que todas las relaciones, directas e indirectas, resultaron significativas. En particular, se destaca la relación directa del autoconcepto académico y las calificaciones de los estudiantes, siendo esta variable la que tiene mayor peso en relación a todas las demás incorporadas al modelo. Este resultado está en línea con otras investigaciones que advierten que el autoconcepto académico sería una de las variables motivacionales con mayor influencia en el rendimiento escolar (Kumar \& Choudhuri, 2017; Núñez et al., 1998; RamosDíaz, Rodríguez-Fernández, Ros \& Antonio-Agirre, 2017). Así, cuando un sujeto se siente competente valora la tarea y favorece los sentimientos de autoeficacia y el control sobre su conducta, se sentiría responsable de sus aprendizajes evidenciando mejores resultados académicos (Morales, 2017; Núñez et al., 1998).

De igual forma, las estrategias de autorregulación tendrían una influencia directa en el rendimiento académico. Esta observación es coherente con otros estudios que señalan que ser un estudiante autorregulado conlleva a obtener mejores logros académicos, principalmente, porque estos alumnos establecen metas, supervisan la ejecución de la tarea, utilizan estrategias efectivas para organizar, codificar y repetir la información a recordar, manejan los recursos en forma efectiva y experimentan orgullo y satisfacción con los resultados de su propio esfuerzo (Gómez \& Romero, 2019; Vera, Poblete \& Díaz, 2019).

Por otra parte, la indisciplina se relaciona directamente, pero en forma negativa, con el rendimiento académico. En palabras de distintos investigadores, este factor tendría repercusiones nocivas en las evaluaciones del alumnado, toda vez que estos se ven enfrentados a comportamientos desadaptativos que imposibilitan o dificultan el aprendizaje (Cerda et al., 2018; Gotzens, Cladellas, Clariana \& Badia, 2015; Proaño-Ponce \& Sánchez-Pazmiño, 2019). Del mismo 
modo, y como era de esperar, las atribuciones causales externas presentan relaciones directas, pero inversas con el rendimiento académico. Estos hallazgos corroboran los resultados de otros estudios que dan cuenta de que los estudiantes con patrones atribucionales básicamente externos obtienen resultados académicos inferiores (Fernández, 2017; Ramudo et al., 2017; Ramudo et al., 2020; Rodríguez-Rodríguez \& Guzmán-Rosquete, 2019).

Los hallazgos del presente estudio resultan coincidentes con los alcanzados en la revisión sistemática de Costa y Fleith (2019) quienes concluyeron que el rendimiento académico está influenciado determinantemente, no solo por factores de tipo cognitivo como la inteligencia o la metacognición, sino también socioemocionales, entre ellos: el autoconcepto, las estrategias de autorregulación y aprendizaje, la autoeficacia, la satisfacción en la escuela y el nivel de compromiso con la tarea. Dicha revisión incluyó estudios que analizaban otras variables como factores demográficos y socioeconómicos, pero centrando su atención en las variables objeto de estudio. De este modo, se puede relevar que las variables socioemocionales funcionarían como un factor de protección que moderaría el efecto del contexto vulnerable o incluso compensaría las consecuencias del mismo (Liu, 2019).

Las interrelaciones directas y moduladas que releva el modelo pueden ser de utilidad para los centros educativos altamente vulnerables, ya que abre espacios para incidir favorablemente en las posibilidades de mejorar los niveles de logros académicos al cautelar algunos elementos desde perspectivas más transversales, o que involucren a toda la comunidad educativa. Así por ejemplo, estos centros podrían fortalecer sus apoyos hacia los estudiantes a través de programas orientados al desarrollo de habilidades cognitivas y motivacionales, desde donde se pueda potenciar y fortalecer el autoconcepto académico y la correcta utilización de estrategias de autorregulación, ya que existe actualmente una serie de herramientas y técnicas para su fortalecimiento (Sanabria, Valencia \& Ibañez, 2017). De la misma forma, se releva como acción importante el anticipar potenciales situaciones de conflicto que lleven al estudiante a presentar conductas desadaptativas en el aula, sobre todo teniendo en cuenta que este factor se relaciona directamente con los logros académicos de los estudiantes.

Finalmente, en cuanto a las proyecciones de este trabajo, la metodología y alcance podrían ser replicados en otras realidades educativas chilenas que presenten diferentes características, como por ejemplo, centros educativos privados o urbanos, o bien estudiantes talentosos, permitiendo analizar si las variables examinadas inciden de la misma manera y con la misma intensidad en los logros académicos de los estudiantes. A modo preliminar, los resultados del estudio en la población escolar chilena llevado a cabo por López et al. (2017), referidos a la interrelación de variables socio-emocionales individuales y de bienestar escolar con el rendimiento académico, controlando los efectos del nivel socioeconómico, sugieren la existencia de dichos efectos diferenciados, lo que 
abre una línea de interés dados los altos índices de estratificación social que se manifiestan en las realidades educativas del país (Bellei, 2013).

Como limitaciones a mencionar sobre el trabajo realizado, se releva naturalmente su característica transversal, la que si bien permite un primer acercamiento al fenómeno, no considera la eventual característica dinámica de las variables socioemocionales consideradas, sobre todo en las etapas de adolescencia, y por lo tanto, restringe de igual manera la efectividad de las posibles propuestas de mejora que se infieran al no contar con un estudio de corte longitudinal, por ejemplo.

Asimismo, si bien el estudio se limita al análisis de factores socioemocionales que pueden influir en el rendimiento académico del alumnado, la literatura muestra que estas variables no actúan de manera aislada y que pueden presentarse ligadas a otras variables de tipo cognitivo como, por ejemplo, la inteligencia o la metacognición, que merecen atención (Costa \& Fleith, 2019).

\section{NOTAS}

Este trabajo de investigación recibió apoyo y financiación de los proyectos Fondecyt 1191064, Proyecto Basal FB0003 del Programa de Investigación Asociativa de CONICYT. 


\section{REFERENCIAS BIBLIOGRÁFICAS}

Agencia de la Calidad de la Educación (2015). Factores asociados a resultados SIMCE e indicadores de desarrollo personal y social 2014. Santiago de Chile.

Alonso, J., \& Sánchez, J. (1992). Estilos atributivos y motivación: El cuestionario EAT. En J. Alonso (Ed.), Motivar en la adolescencia: Teoría, evaluación $e$ intervención (pp. 39-81). Servicio de Publicaciones de la Universidad Autónoma.

Amata, M., Di Blasi, F., Cantagallo, C., Costanzo, A., Finocchiaro, M., Zuccarello, R., Di Nuovo, S., \& Buono, S. (2017) Stile attributivo, rappresentazione dell'intelligenza e obiettivi di apprendimento in ragazzi con Funzionamento Intellettivo Borderline. Psicologia Clinica dello Sviluppo, 2, 291308. https://doi.org/10.1449/87539

Arias, R., \& Aparicio, A. (2020). Conciencia metacognitiva en ingresantes universitarios de ingeniería, arquitectura y ciencias aeronáuticas. Propósitos y Representaciones, 8(1). http://dx.doi. org/10.20511/pyr2020.v8n1.272

Barca-Lozano, A., Montes-Oca-Báez, G., \& Moreta, Y. (2019). Motivación, enfoques de aprendizaje y rendimiento académico: impacto de metas académicas y atribuciones causales en estudiantes universitarios de educación de la República Dominicana. Revista Caribeña de Investigación Educativa, 3(1), 19-48. https://doi.org/10.32541/recie.2019.v3i1. pp19-48

Bellei, C. (2013). El estudio de la segregación socioeconómica y académica de la educación chilena. Estudios Pedagógicos, 39(1), 325-345. https://doi. org/10.4067/S0718-07052013000100019

Bravo, M., Salvo, S., Miranda, H., \& Bangdiwala (2018). What school social climate factors affect mathematics performance in secondary school students? A multilevel análisis. Culture and Education, 132(2), 506-528. https:// doi.org/10.1080/11356405.2020.1785138

Castrillón, E., Morillo, S., \& Restrepo, L. (2020). Diseño y aplicación de estrategias metacognitivas para mejorar la comprensión lectora en estudiantes de secundaria. Ciencias Sociales y Educación, 9(17), 203-231.

Cazalla-Luna, N., \& Molero, D. (2015), Revisión teórica sobre el autoconcepto y su importancia en la adolescencia. Revista Electrónica de Investigación y Docencia (REID), 10, 43-64.

Cerda, G., \& Vera-Sagredo, A. (2019). Rendimiento en matemáticas: Rol de distintas variables cognitivas y emocionales, su efecto diferencial en función del sexo de los estudiantes en contextos vulnerables. Revista Complutense de Educación, 30(2), 1-16. https://doi.org/10.5209/RCED.57389

Cerda, G., Pérez, C., Aguilar, M., \& Aragón, E. (2018). Algunos factores asociados al desempeño académico en matemáticas y sus proyecciones en la formación docente. Educação e Pesquisa, 44, 1-19. https://doi.org/10.1590/S16784634201706155233

Cerda, G., Pérez, C., Elipe, P., Casas, J. A., \& Del Rey, R. (2019). Convivencia escolar y su relación con el rendimiento académico en alumnado de Educación Primaria. Revista de Psicodidáctica, 24(1), 46-52. https://doi.org/10.1016/j. psicod.2018.05.001.

Cerda, G., Salazar, O., Cerda, K., \& Riffo, B. (2019) Habilidad lectora, estilos atribucionales, estrategias cognitivas y de autorregulación, inteligencia lógica: Predictores del rendimiento en académico en lenguaje de estudiantes de establecimientos educativos de alta vulnerabilidad social. Onomázein 45, 31-57. https://doi.org/10.7764/ onomazein. 45.01 
ANGÉLICA VERA SAGREDO, GAMAL CERDA ETCHEPARE, ESTÍBALIZ ARAGÓN MENDIZÁBAL, CARLOS PÉREZ WILSON

Cerda, G., Salazar, Y., Guzmán, C., \& Narváez, G. (2018). Impacto de la convivencia escolar sobre el rendimiento académico, desde la percepción de estudiantes con desarrollo típico y necesidades educativas especiales. Propósitos y Representaciones, 6(1), 247300. https://doi.org/10.20511/pyr2018. v6n1.194.

Chacón, F., \& Huertas, C. (2017). The causes of school failure in secondary school students: validation of a psychosocial model with structural equations. The Spanish Journal of Psychology, 20, 1-13. https://doi.org/10.1017/sjp.2017.60.

Claver, F., Martínez-Aranda, L., Conejero, M., \& Gil-Arias, A. (2020). Motivation, discipline, and academic performance in physical education: A holistic approach from achievement goal and self-determination theories. Frontiers in Psychology, 11, 1-11. https://doi. org/10.3389/fpsyg.2020.01808.

Costa, B. C. G., \& Fleith, D. D. S. (2019). Prediction of academic achievement by cognitive and socio-emotional variables: a systematic review of literature. Trends in Psychology, 27(4), 977-991. https://doi. org/10.9788/TP2019.4-11

Daily, S., Mann, M., Lilly, C., Dyer, A., Smith, M., \& Kristjansson, A. (2020). School climate as an intervention to reduce academic failure and educate the whole child: A longitudinal study. Journal of School Health, 90(3), 182-193. https://doi.org/10.1111/josh.12863

Del Rey, R., Casas, J., \& Ortega, R. (2017). Desarrollo y validación de una escala de Convivencia Escolar (ECE). Universitas Psychologica, 16(1), 275-285. https://doi. org/10.11144/Javeriana.upsy16-1.dvec

Delgado, B., Aparisi, F., García-Fernández, J., Redondo, J., \& Inglés, C. (2018). Estilos atribucionales en estudiantes españoles de Educación Secundaria Obligatoria con alta ansiedad social autoinformada. Revista Latinoamericana de Psicología, 50(2), 89-97. http://dx.doi. org/10.14349/rlp.2018.v50.n2.2

Educación 2020 (2015). Cómo la convivencia escolar mejora los aprendizajes de niños y niñas. https:// n9.cl/15nq

Educación 2020 (s.f.). Lo que Usted debe saber si quiere hablar de educación. https://n9.cl/yqk52

Elipe, P., Casas, J. A., Rey, R. del, OrtegaRuiz, R., Cerda, G., \& Pérez, C. (2018). Validation and cross-cultural robustness of the School-wide Climate Scale (SCS) across Spanish and Chilean students. Studies in Educational Evaluation, 56, 182-188. https://doi.org/10.1016/j. stueduc.2018.01.002

Fernández, A. (2017). Motivación y rendimiento académico en la universidad. Ministerio de Educación Superior, Ciencia y Tecnología.

García, B. (2001). Cuestionario de Autoconcepto GARLEY (CAG). Versión 1.0. Instituto de Orientación Psicológica EOS.

García-Ripa, M., Sánchez-García, M., \& Risquez, A. (2016). Estrategias de aprendizaje y autorregulación motivacional. Identificación de perfiles para la orientación de estudiantes universitarios de nuevo ingreso. RIDEP, Revista Iberoamericana de Diagnóstico y Evaluación - e Avaliação Psicológica, 1(41), 39-57.

Gómez, J., \& Romero, A. (2019). Enfoques de aprendizaje, autorregulación y autoeficacia y su influencia en el rendimiento académico en estudiantes universitarios de Psicología. European Journal of Investigation in Health, Psychology and Education, 9(2), 95-107. https://doi.org/10.30552/ejihpe.v9i2.323

González, M., Leal, D., Segovia, C., \& Arancibia, V. (2012). Autoconcepto y talento: una relación que favorece el logro académico. Psykhe, 21(1), 37-53. 
González-Berruga, M.Á. (2020). Sistemas de atribución causal sobre el fracaso escolar de estudiantes y docentes en la Enseñanza Secundaria Obligatoria. Revista Científica Hallazgos 21, 5(1), 5770.

Gonzálvez, C., Inglés, C., Vicent, M., Lagos-San Martín, N., Sanmartín, R., \& García-Fernández, J. (2016). Diferencias en ansiedad escolar y autoconcepto en adolescentes chilenos. Acta de investigación psicológica, 6(3), 2509-2515. https://doi.org/10.1016/j. aipprr.2016.08.002

Gotzens, C., Cladellas, R., Clariana, M., \& Badia, M. (2015). Indisciplina instruccional y convencional: $\mathrm{Su}$ predicción en el rendimiento académico. Revista Colombiana de Psicología, 24(2), 317-330. https://doi.org/10.15446/rcp. v24n2.44148.

Hernández, P., \& García, L. (1995). Cuestionario de Estrategias de Control en el Estudio (ECE). Departamento de Psicología Educativa, Evolutiva y Psicobiología, Universidad de La Laguna.

Huang, C., Yu, C., \& Wu, I. (2019). Relationships between the parentchild interaction, self-concept, and school adjustment of junior high school students with disabilities. Journal of Research in Education Sciences, 63(1), 103-140. https://doi.org/10.6209/ JORIES.2018.63(1).04

Ibarra, J. (2019). Estilos atribucionales en la percepción de logro académico y de relaciones interpersonales en estudiantes de pregrado de psicología. Revista de Investigación Psicológica, (21), 73-86.

Ibrahim, H., \& Johnson, O. (2020), School discipline, race-gender and STEM readiness: A hierarchical analysis of the impact of school discipline on Math achievement in high school. Urban Review, 52(1), 75-99. https://doi. org/10.1007/s11256-019-00513-6.
Jurado, P., Blanco, H., Zueck, M., \& Peinado, J. (2016). Composición factorial del cuestionario de estilos atributivos área de logros académicos en universitarios mexicanos. Formación Universitaria, 9(6), 83-92.

Krskova, H., \& Baumann, C. (2017), School discipline, investment, competitiveness and mediating educational performance. International Journal of Educational Management, 31(3), 293-319. https://doi. org/10.1108/IJEM-05-2016-0099.

Kumar, S., \& Choudhuri, R. (2017), Academic self concept and academic achievement of secondary school students. American Journal of Educational Research, 5(10), 1108-1113, https://doi. org/10.12691/education-5-10-13.

Lagos, N., Inglés, C., Ossa, C., GonzálezMaciá, C., Vicent-Juan, M., \& GarcíaFernández, J. (2016). Relación entre atribuciones de éxito y fracaso académico y ansiedad escolar en estudiantes chilenos de educación secundaria. Psicologías desde el Caribe, 33(2), 146157.

Liu, A. (2019). Can non-cognitive skills compensate for background disadvantage?-the moderation of noncognitive skills on family socioeconomic status and achievement during early childhood and early adolescence. Social science research, 83, 3-22. https://doi. org/10.1016/j.ssresearch.2019.04.019

López, V., Oyanedel, J., Bilbao, M., Torres, J., Oyarzún, D., Morales, M., Ascorra, P. \& Carrasco, C. (2017). School achievement and performance in Chilean high schools: The mediating role of subjective wellbeing in school-related evaluations. Frontiers in Psychology, 8, 1-15, https:// doi.org/10.3389/fpsyg.2017.01189.

Mardia, K., Kent, T., \& Bibby, J. (2003). Multivariate Analysis. Academic Press.

Martínez, J., \& Medina, A. (2019). Enfoques de aprendizaje, autorregulación y autoeficacia y su influencia en el 
rendimiento académico en estudiantes universitarios de Psicología. European Journal of Investigation in Health, Psychology and Education, 9(2), 95-107. https://doi.org/10.30552/ejihpe.v9i2.323.

Martínez, M., \& Valiente, C. (2019). Autorregulación afectivo- motivacional, resolución de problemas y rendimiento matemático en Educación Primaria. Educatio Siglo XXI, 37(3), 33-54. https:// doi.org/10.6018/educatio.399151

Ministerio de Educación (2015). Convivencia escolar y resolución de conflictos. Material de apoyo para profesoras y profesores. Nuestros Temas, $\mathrm{N}^{\mathrm{o}}$ 30. Gobierno de Chile https://n9.cl/ imi3

Ministerio de Educación (2020). Coordinación Nacional de Educación de Personas Jóvenes y Adultas (EPJA). División de Educación General. https:// n9.cl/ew4vp

Morales, F. (2017). Relaciones entre afrontamiento del estrés cotidiano, autoconcepto, habilidades sociales e inteligencia emocional. European Journal of Education and Psychology, 10(2), 41-48. https://doi.org/10.1016/j. ejeps.2017.04.001.

Morales, M., \& Morales, M. (2019). Adolescentes de buen rendimiento escolar de colegios vulnerables y la resignificación de su experiencia escolar. Integración Académica en Psicología, $7(20), 32-45$

Ngwokabuenui, P. Y. (2015). Students' indiscipline: Types, causes and possible solutions: The case of secondary schools in Cameroon. Journal of Education and Practice, 6(22), 64-72.

Núñez, J., González-Pienda, J., García, M., González-Pumariega, S., Roces, C., Álvarez, L., \& González, M. (1998). Estrategias de aprendizaje, autoconcepto y rendimiento académico. Psicothema, 10(1), 97- 109.
Orellana, V., Canales, M., Bellei, C., \& Guajardo, F. (2019). Individuación y mercado educacional en Chile. Revista Brasileira de Politica e administracao da educacao, RBPAE, 35(1), 141-157.

Padua, L. (2019), Factores individuales y familiares asociados al bajo rendimiento académico en estudiantes universitarios. Revista mexicana de investigación educativa, RMIE, 24(80), 173-195.

Proaño-Ponce, W. P., \& Sánchez-Pazmiño, I. C. (2019). Rendimiento académico, una reflexión desde la conducta en estudiantes de un colegio de Guayaquil, Ecuador. Avances, 21(3), 305-318.

Ramos-Díaz, E., Rodríguez-Fernández, A., Ros, I., \& Antonio-Agirre, I. (2017). Implicación escolar y autoconcepto multidimensional en una muestra de estudiantes españoles de secundaria. Revista Complutense de Educación, 28(4), 1103-1118. https://doi.org/10.5209/ RCED.51600.

Ramudo, I., Barca, A., Brenlla, J. C., \& Barca, E. (2017). Metas académicas, atribuciones causales y género: su determinación en el rendimiento académico del alumnado de bachillerato. Revista de estudios e investigación en Psicología y Educación, 1. https://doi. org/1017979/reipe.20170.01.2436

Ramudo, I., Barca, E., Brenlla, J. C., Peralbo, M., \& Barca A. (2020). Predicción del rendimiento académico del alumnado de Bachillerato: efecto de los enfoques de aprendizaje y atribuciones causales. Revista de Psicología y Educación, 15(2), 108-120. https://doi.org/10.23923/rpye2020.02.190

Rodríguez-Rodríguez, D., \& GuzmánRosquete, R. (2019). Rendimiento académico de adolescentes declarados en situación de riesgo. Revista de Investigación Educativa, 37(1), 147-162. https://doi.org/.6018/rie.37.1.303391. 
Rosas, R., \& Santa Cruz, C. (2013). Dime en qué colegio estudiaste y te diré qué CI tienes. Ediciones UC. Chile.

Ruiz, P. (2017). La influencia del estilo de atribución interna en la autoestima. Revista Publicando, 10(1), 358-366.

Sanabria, L., Valencia, N., \& Ibañez, J. (2017). Efecto del entrenamiento en autorregulación para el aprendizaje de la matemática. Praxis \& Saber, 8(16), 35-56.

Suárez, J., Fernández, A., Rubio, V., \& Zamora, Á. (2015). Incidencia de las estrategias motivacionales de valor sobre las estrategias cognitivas y metacognitivas en estudiantes de secundaria. Revista Complutense de Educación, 27(2), 421-435. https:// doi.org/10.5209/rev_RCED.2016.v27. n2.46329.

Valle, A., Rodríguez, S., Cabanach, R., Núñez, J., González-Pineda, A., \& Rosário, P. (2009). Diferencias en rendimiento académico según los niveles de las estrategias cognitivas y de las estrategias de autorregulación. Suma Psicológica, 6(2), 31-42.

Vengas, S., Garay, M., \& Sillard, M. (2016). La ley SEP en las escuelas de Punta Arenas, entre los años 2008-2015. Sophia Austral, 18(2), 95-109.
Vera, A., Cerda, G., \& Melipillán, R. (En prensa). Adaptación de la escala EAT en la población escolar chilena. Revista Estudios Pedagógicos.

Vera, A., Poblete, S., \& Díaz, C. (2019). Percepción de estrategias y estilos de aprendizaje en estudiantes universitarios de primer año. Revista Cubana de Educación Superior, 38(1), 1-23.

Villalta, M. (2010). Factores de resiliencia asociados al rendimiento académico en estudiantes de contextos de alta vulnerabilidad social. Revista de Pedagogía, 31(88), 159-188.

Villalta, M., \& Saavedra, E. (2012). Cultura escolar, prácticas de enseñanza y resiliencia en alumnos y profesores de contextos sociales vulnerables. Universitas Psychologica, 11(1), 67-78.

Wang, W., Vaillancourt, T., Brittain, H. L., McDougall, P., Krygsman, A., Smith, D., Cunningham, C. E., Haltigan, J. D., \& Hymel, S. (2014). School climate, peer victimization, and academic achievement: Results from a multiinformant study. School Psychology Quarterly, 29(3), 360-377, https://doi. org/10.1037/spq0000084

Weiner, B. (1979). A theory of motivation for some classroom experiences. Journal of Educational Psychology, 71(1), 3-25. 


\section{PERFIL ACADÉMICO Y PROFESIONAL DE LOS AUTORES}

Angélica Vera Sagredo. ORCID: https://orcid.org/0000-0003-1657-2241

Angélica Vera Sagredo es doctora en Educación, profesora de la Universidad Católica de la Santísima Concepción, Chile. Sus líneas de investigación se centran en el área de la cognición y factores asociados al aprendizaje, innovación en educación e implementación de Tecnologías de la Información y Comunicación en educación. Email: avera@ucsc.cl

Gamal Cerda Etchepare. ORCID: https://orcid.org/0000-0002-3662-4179

Gamal Cerda Etchepare es profesor Asociado de la Facultad de Educación de la Universidad de Concepción, Chile. Sus líneas de investigación se centran en el área de la cognición y factores asociados al aprendizaje, principalmente en el ámbito de las matemáticas, resolución de problemas, inteligencia lógica y competencias matemáticas tempranas.

Email: gamal.cerda@udec.cl

Estíbaliz Aragón Mendizábal. ORCID: https://orcid.org/0000-0002-0440-5705

Estíbaliz Aragón Mendizábal es doctora en Ciencias Sociales y Jurídicas. Actualmente, es Profesora Ayudante Doctora en el Departamento de Psicología de la Universidad de Cádiz. Ha participado como investigadora en proyectos $\mathrm{I}+\mathrm{D}+\mathrm{i}$ nacionales e internacionales y ha realizado numerosas publicaciones en materia de aprendizaje matemático temprano y cognición matemática. Asimismo, ha formado parte como investigadora en otros proyectos y publicaciones relacionados con el aprendizaje en Educación Infantil y Educación Superior. Email: estivaliz.aragon@uca.es

Carlos Pérez Wilson. ORCID: https://orcid.org/0000-0001-6035-7341

Carlos Pérez Wilson. Profesor del Instituto de Ciencias Sociales de la Universidad de O’Higgins, Chile. Sus líneas de investigación se centran principalmente en el enriquecimiento de contenidos matemáticos sobre el currículum chileno, el impacto de la metodología $\mathrm{ABN}$, y la modelización de la interacción entre variables educativas y el efecto del enriquecimiento matemático sobre éstas. Email: carlos.perez@uoh.cl

Fecha Recepción del Artículo: 14. Septiembre. 2020

Fecha Modificación del Artículo: 21. Enero. 2021

Fecha Aceptación del Artículo: 03. Febrero. 2021

Fecha Revisión para Publicación: 25. Febrero. 2021 
